\title{
Characteristics of seismic activity before and after the 2018 M6.7 Hokkaido Eastern Iburi earthquake
}

\author{
Takao Kumazawa ${ }^{1 *}$, Yosihiko Ogata $^{2}$ and Hiroshi Tsuruoka ${ }^{1}$
}

\begin{abstract}
We applied the epidemic type aftershock sequence (ETAS) model, the two-stage ETAS model and the non-stationary ETAS model to investigate the detailed features of the series of earthquake occurrences before and after the M6.7 Hokkaido Eastern Iburi earthquake on 6 September 2018, based on earthquake data from October 1997. First, after the 2003 M8.0 Tokachi-Oki earthquake, seismic activity in the Eastern Iburi region reduced relative to the ETAS model. During this period, the depth ranges of the seismicity were migrating towards shallow depths, where a swarm cluster, including a M5.1 earthquake, finally occurred in the deepest part of the range. This swarm activity was well described by the non-stationary ETAS model until the M6.7 main shock. The aftershocks of the M6.7 earthquake obeyed the ETAS model until the M5.8 largest aftershock, except for a period of several days when small, swarm-like activity was found at the southern end of the aftershock region. However, when we focus on the medium and larger aftershocks, we observed quiescence relative to the ETAS model from 8.6 days after the main shock until the M5.8 largest aftershock. For micro-earthquakes, we further studied the separated aftershock sequences in the naturally divided aftershock volumes. We found that the temporal changes in the background rate and triggering coefficient (aftershock productivity) in respective sub-volumes were in contrast with each other. In particular, relative quiescence was seen in the northern deep zones that includes the M5.8 largest aftershock. Furthermore, changes in the $b$-values of the whole aftershock activity showed an increasing trend with respect to the logarithm of elapsed time during the entire aftershock period, which is ultimately explained by the spatially different characteristics of the aftershocks.
\end{abstract}

Keywords: ABIC, AIC, Bayesian method, b-Values, Stationary ETAS model, Two-stage ETAS model, Non-stationary ETAS model

\section{Background}

The epidemic type aftershock sequence (ETAS) model (Ogata 1988) provides a good fit to standard earthquake occurrences. However, there are non-standard earthquake series that appear under transient stress changes caused by external seismic or internal aseismic forces such as slow slips or fluid intrusions (Terakawa et al. 2013; Terakawa 2014; Yoshida et al. 2017, 2018a, b). These events trigger transient non-standard earthquake swarms, and they are poorly fitted by the stationary ETAS model (Kumazawa and Ogata 2013; Kumazawa

\footnotetext{
*Correspondence: tkuma@eri.u-tokyo.ac.jp

${ }^{1}$ Earthquake Research Institute, University of Tokyo, Tokyo, Japan Full list of author information is available at the end of the article
}

et al. 2017a, b). In this study, we examine non-stationary extensions of the ETAS model that cover non-standard cases. These models allow the parameters to be timedependent and can be estimated by the empirical Bayes method. The best model is selected among the competing models to provide the inversion solutions of non-stationary changes. As a case study we investigated seismic activity in the Hokkaido Eastern Iburi region, before and after the M6.7 earthquake on September 16, 2018, based on a series earthquake occurrence data after October 1997.

On September 6, 2018, a M6.7 earthquake occurred at a depth of $37 \mathrm{~km}$ in the Mid-eastern part of the Iburi region in Hokkaido, Japan. This earthquake occurred within the plate of the land (North American plate). The 
Table 1 Hypocenter coordinates and focal mechanisms of the target earthquakes

\begin{tabular}{lllllllll}
\hline Event & Occurrence time & Mag. & Lat. $\left(^{\circ}\right)$ & Lon. $\left(^{\circ}\right)$ & Dep. $(\mathbf{k m})$ & Strike & Dip & Rake \\
\hline (i) & 26-Sep-03, 04:50:07.64 & 8.0 & 41.7797 & 144.0785 & 42. & $31 ; 249$ & $78 ; 15$ & $81 ; 127$ \\
(ii) & 1-Jul-17, 23:45:52.92 & 5.1 & 42.7877 & 141.859 & 27.47 & $159 ; 20$ & $40 ; 58$ & $56 ; 115$ \\
(iii) & 6-Sep-18, 03:07:59.33 & 6.7 & 42.6908 & 142.0067 & 37.04 & $134 ; 349$ & $30 ; 65$ & $59 ; 107$ \\
(iv) & 21-Feb-19, 21:22:40 & 5.8 & 42.7662 & 142.0033 & 33.3 & $131 ; 350$ & $24 ; 71$ & $54 ; 105$ \\
\hline
\end{tabular}

Listed are (i) the 2003 M8.0 Tokachi-Oki earthquake, (ii) an off fault M5.1 pre-shock, (iii) the M6.7 main shock, and (iv) its largest aftershock

earthquake exhibited a reverse fault type focal mechanism with a pressure axis in the ENE-WSW direction. The earthquake occurred near the major active faults (the Ishikari Lowland Faults), but at greater depths (Earthquake Research Committee 2010). From the crustal deformation caused by the M6.7 earthquake observed by SAR and GNSS, estimations suggested that a reverse fault motion in a high angle fault plane with an almost north-south trend had occurred; the upper end of the ruptured fault was located at a depth of about $16 \mathrm{~km}$ and its moment magnitude was estimated to be $M w 6.6$ (Geographical Survey Institute 2018). See also Kobayashi et al. (2019) in the current special issue.

Fourteen months before this main shock, a swarm cluster occurred, followed immediately by a M5.1 earthquake and subsequent aftershocks near the focal zone of the main shock.

The aftershock activity of the 2018 Eastern Iburi earthquake occurred predominantly within an area approximately $30 \mathrm{~km}$ in length that extended in a north-south direction. The time decay of this activity shows various characteristics that will be discussed in this paper. On February 21 2019, a M5.8 earthquake occurred at a depth of about $35 \mathrm{~km}$, in the northern part of the primary aftershock zone. The focal mechanism of the earthquake was marked by a pressure axis in the east-west direction.

Table 1 summarizes the hypocenter data and the mechanisms of the F-net catalog of National Research Institute for Earth Science and Disaster Resilience (2019) of the above-mentioned conspicuous earthquakes as supplied by the Japan Meteorological Agency (JMA). In this work, we analyze seismic activities in detail, including those associated with such conspicuous earthquakes, by using statistical models and methods that are summarized in the next section.

\section{Models and methods}

\section{The reference ETAS model}

The ETAS model (Ogata 1988, 1989; Utsu et al. 1995) is a point-process model representing the activity of earthquakes of magnitude $M_{c}$ and above in a certain region during a certain time interval. The seismic activity includes the background activity at a constant occurrence rate $\mu$ (Poisson process). The model assumes that each earthquake (including the aftershock of another earthquake) is followed by aftershocks, although only aftershocks of magnitude $M_{c}$ and larger are included in the data. The aftershock activity is represented by the Omori-Utsu formula (Utsu 1961) in the time domain. The rate of aftershock occurrence at time $t$ following the $i$ th earthquake (time: $t i$, magnitude: $M i$ ) is given by

$$
v_{i}(t)=K_{0} e^{\alpha\left(M_{i}-M_{c}\right)}\left(t-t_{i}+c\right)^{-p}, \quad \text { for } t>t_{i},
$$

where $K_{0}, \alpha, c$, and $p$, are constants, which are common to each target aftershock sequence in a region. The rate of occurrence of the whole earthquake series at time $t$ becomes

$$
\lambda\left(t \mid H_{t}\right)=\mu+\sum_{S<t_{i}<t} v_{i}(t) .
$$

The summation is performed for all $i$ satisfying $t i<t$. Here, $H_{t}$ represents the history of occurrence times with associated magnitudes from the data $\left\{\left(t_{i}, M_{i}\right)\right\}$ before time $t$. The parameter set $\theta=(\mu, K, c, \alpha, p)$ represents characteristics of the seismic activity of a focal region; hereafter, the unit of the parameters are 'event number/day', 'event number/day', 'day', 'magnitude ${ }^{-1}$, and no dimension', respectively. In this manuscript, we are particularly concerned with the parameters $\mu$ (the background rate) and $K_{0}$ (the triggering coefficient). See Additional file 1: S1 for more details.

We estimate the parameters using the maximum-likelihood method where the log-likelihood function,

$$
\ln L(\theta ; S, T)=\sum_{\left\{i: S<t_{i}<T\right\}} \ln \lambda_{\theta}\left(t_{i} \mid H_{t_{i}}\right)-\int_{S}^{T} \ln \lambda_{\theta}\left(t \mid H_{t}\right) \mathrm{d} t,
$$

is maximized with respect to the parameters $\theta=(\mu, K, c$, $\alpha, p)$, and we call such an estimate the maximum-likelihood estimate (MLE), denoted by $\hat{\theta}=\left(\hat{\mu}, \hat{K}_{0}, \hat{c}, \hat{\alpha}, \hat{p}\right)$. Here, $S$ is the starting time for the analysis, and $\left\{\left(t_{i}, M_{i}\right)\right.$, $\left.M_{i} \geq M_{c} ; i=1,2, \ldots\right\}$ are the occurrence times and magnitudes of earthquakes in a time interval $\left[0, T_{\text {end }}\right]$, which is divided by a precursory time interval $[0, S]$ and a target 
time interval $[S, T]$ for the fitting, and a prediction interval $\left[T, T_{\text {end }}\right]$. its integral function $\Lambda_{\hat{\theta}}\left(S, t \mid H_{S}\right)$ of $t$ is closely overlap the observed function of the cumulative counts $N(t)$ of

$$
\begin{aligned}
& \text { Precursory interval Target interval Prediction interval }
\end{aligned}
$$

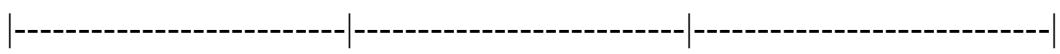

$$
\begin{aligned}
& \begin{array}{llll}
0 & S & T & T_{\text {end }}
\end{array} \\
& \text { ( } \left.\begin{array}{lll}
\text { zts } & \text { zte } & \text { ztend }
\end{array}\right)
\end{aligned}
$$

The precursory period is necessary to avoid biased estimations because the ETAS model is history-dependent. Note, for example, in the case where we fit ETAS model to an aftershock sequence, the data in the target interval $[S, T]$ in the log-likelihood (3) are required detection completeness; but the aftershocks immediately followed after the large main shock are imperfectly detected. On the otherhand, we need to set in the precursory period $[0, S)$ because the main shock and such large aftershocks are very influential to the occurrences of aftershocks in the target period; namely, $\lambda_{\theta}\left(t \mid H_{t}\right)$ always includes the data of the main shock and aftershocks during the period $[0, S)$ as the precursory history. Even for the case of general seismic activity, we need a suitable precursory period in the very beginning of the available occurrence data.

In Supplemental Information of this manuscript (abbreviated by SI, hereafter), the respective MLEs are listed for each target time interval $[S, T]$ where $S$ and $T$ indicated in the tables stands for "tstart" and "zte" in the notation of (4), respectively, those in (4) are variables set in the FORTRAN package SASeis2006 (Ogata 2006a, b), and the X-window-based interactive graphical software XETAS (Tsuruoka and Ogata 2015a, b; Ogata and Tsuruoka 2016). Again, in fitting the ETAS model, earthquake data in the target and prediction interval should be completely detected above the cut-off magnitude of $M_{c}$.

Then, using the MLE, we can visualize how well or how poorly the model fits to the earthquake sequence by comparing the cumulative number $N(S, t)$ of earthquakes with the rate calculated by the model

$$
\Lambda_{\hat{\theta}}(S, t)=\int_{S}^{t} \lambda_{\hat{\theta}}\left(u \mid H_{u}\right) d u
$$

for the time interval $S<t<T$. If the model presents a good approximation of observed seismicity, we can expect that the events. Furthermore, plots of $N(t)$ against the transformed time $\tau=\Lambda_{\hat{\theta}}\left(S, t \mid H_{S}\right)$ shows nearly straight line. See Additional file 1: S2 for more details.

\section{Two-stage ETAS model and change-point problem}

When the stationary ETAS model (2) does not fit a dataset well, the simplest alternative model is a "two-stage ETAS model" that takes different parameter values in sub-periods before and after a particular time, called a "change-point". In change-point analysis, we divide the whole period into two disjoint periods to fit the ETAS models separately (two-stage ETAS model). This is one of the ways to treat the non-stationary data and can be best applied to the cases where there is a clear-cut time instant across which the ETAS parameters change. We occasionally observe such a change-point when a notably large earthquake or slow slip event (regardless of observed or unobserved) occur in or near the target region. Many of the preceding works (e.g., Ogata et al. 2003; Ogata 2005, 2006a, 2007, 2010; Kumazawa et al. 2010) adopted this method to their case studies, and the details can be found therein.

Testing whether or not the seismicity pattern changes at a time $T_{0}$ in a given period $[S, T]$ is reduced to a problem of model selection method by using the Akaike information criterion (AIC; Akaike 1973, 1974, 1977). In this procedure, we separately fit the ETAS models on the divided periods $\left[S, T_{0}\right]$ and $\left[T_{0}, T\right]$, then compare their total goodness-of-fit against the one fitted over the whole period $[S, T]$ by the minimum AIC principle as follows:

Let $\mathrm{AIC}_{0}$ be the AIC of the stationary ETAS model estimated on the whole period $[S, T], \mathrm{AIC}_{1}$ be that on the first period $\left[S, T_{0}\right]$ and $\mathrm{AIC}_{2}$ on the second period $\left[T_{0} T\right]$ where the data in the first period is used for the precursory period, then 


$$
\begin{aligned}
& \mathrm{AIC}_{0}=-2 \max _{\theta_{0}} \ln L\left(\theta_{0} ; S, T\right)+2 \operatorname{dim} \theta_{0} \\
& \mathrm{AIC}_{1}=-2 \max _{\theta_{1}} \ln L\left(\theta_{1} ; S, T_{0}\right)+2 \operatorname{dim} \theta_{1} \\
& \mathrm{AIC}_{2}=-2 \max _{\theta_{2}} \ln L\left(\theta_{2} ; T_{0}, T\right)+2 \operatorname{dim} \theta_{2},
\end{aligned}
$$

where $\operatorname{dim} \theta_{0}, \operatorname{dim} \theta_{1}$ and $\operatorname{dim} \theta_{2}$ are the number of adjusted parameters, respectively.

If a change-point $T_{0}$ is hypothetically prefixed based on some information other than the occurrence data themselves, such as a notable geophysical event or a notable outside large earthquake, then we can compare $\mathrm{AIC}_{0}$ with $\mathrm{AIC}_{1}+\mathrm{AIC}_{2}$ to select the model with the smaller value, which performs better fit to the data in the entire target period $[S, T]$. Here, it is useful to remember that, if the difference $\triangle \mathrm{AIC}=\mathrm{AIC}_{1}+\mathrm{AIC}_{2}-\mathrm{AIC}_{0}$ takes negative value, the quantity $\exp \{-0.5 \Delta \mathrm{AIC}\}$ indicates the relative probability how the two-stage model outperform the single stationary ETAS model (Akaike 1980).

On the other hand, if the change-point $T_{0}$ is searched from target data, the two-stage model becomes harder to be accepted. Namely, $\mathrm{AIC}_{12}$ as the AIC of the two-stage ETAS model should be calculated as follows:

$$
\mathrm{AIC}_{12}=\mathrm{AIC}_{1}+\mathrm{AIC}_{2}+2 q ;
$$

here the additional term $q$ is the degree of freedom to search for the best candidate $T_{0}$ (MLE of a changepoint) from the data. The value of $q$ depends on sample size (number $n$ of earthquakes in the target period $[S$, $T]$ ), ranging 4-5 monotonically for $n$ between 100 and 1000 (Ogata 1992, 1999; Kumazawa et al. 2010; Bansal and Ogata 2013). This penalty term $q$ in addition to the increased number of parameters imposes a hurdlefor the two-stage ETAS model to be selected. See Additional file 1: S3 for more details.

\section{Theoretical cumulative function and the time transformation}

Suppose that the MLE $\hat{\theta}=\left(\hat{\mu}, \hat{K}_{0}, \hat{c}, \hat{\alpha}, \hat{p}\right)$ of the ETAS model in the first period $\left[S, T_{0}\right]$ are given. Then the integral (3) extended over the time interval $\left[T_{o} t\right]$ provides the expected cumulative number of earthquakes on the extended period. We consider time transformation

$$
\tau=\Lambda_{\hat{\theta}}\left(T_{0}, t\right)
$$

from $t$ to $t$, which transforms the ordinary-time sequence of earthquakes; $\left(t_{1}, t_{2}, \ldots, \mathrm{t}_{N}\right)$ into the transformed-time sequence $\left(\tau_{1}, \tau_{2}, \ldots, \mathbf{\tau}_{N}\right)$ in the time interval $\left[0, \Lambda\left(T_{0}, T\right)\right]$, which we call the residual point process (RPP). If the sequence continues in the remaining period, we expect the integrated function (3) and the empirical cumulative counts $N(t)-N\left(T_{0}\right)$ of the observed earthquakes to be close to each other. This implies that the transformed sequence, or RPP, $\left(\tau_{1}, \tau_{2}, \ldots ., \tau_{N}\right)$ in the time interval $[0$, $\Lambda\left(T_{0}, T\right)$ ] appears to be a stationary Poisson process (uniformly distributed occurrence times) with unit intensity (occurrence rate), but it deviates upward or downward otherwise.

Let $\tau_{0}=\Lambda\left(T_{0}\right)$, and $N\left(\tau_{0}, \tau\right)$ be the number of events in the interval $\left(\tau_{0}, \tau\right)$ in the transformed time axis. If no change occurs in aftershock activity, the extrapolated RPP is the standard stationary Poisson process with the same unit intensity, and the deviation $N\left(\tau_{0}, \tau\right)-\left(\tau-\tau_{0}\right)$ of the empirical cumulative function is approximately distributed according to the normal distribution $N(0, \Delta \tau)$, where $\Delta \tau=\tau-\tau_{0}$. However, when the number of events $N\left(\sigma, \tau_{0}\right)$ in the target interval $\left(\sigma, \tau_{0}\right)$ is not large enough, the significance actually depends on the sample size, due to the estimation accuracy of the parameters for the transformation. Thus, taking such accuracy into consideration, the error distribution of $\Delta N\left(\tau_{0}, \tau\right)$ is modified to be $N\left(0, \Delta \tau+(\Delta \tau)^{2} / N\left(\sigma, \tau_{0}\right)\right)$, derivation evaluation of which can be seen in Ogata (1992). Hereafter, we define the significance of deviations in the case where the empirical cumulative function deviates outside the parabola of $95 \%$ significance $2\left\{\Delta \tau+(\Delta \tau)^{2} / N\left(\sigma, \tau_{0}\right)\right\}$.

The integral over a fixed period of the conditional intensity function gives the expected number of earthquakes. On this basis, we focus on instances in which the number of earthquakes in an area is significantly less or larger than the extension of the fitted ETAS model, which we call "relative quiescence" and "relative activation", respectively.

\section{Non-stationary ETAS models}

The stationary ETAS model in (2) is temporally extended to the non-stationary ETAS model (Kumazawa and Ogata 2013; Kumazawa et al. 2017a, b) in the following form:

$$
\lambda\left(t \mid H_{t}\right)=\mu(t)+\sum_{t_{i}<t} K_{0}\left(t_{i}\right) e^{\alpha\left(M_{i}-M_{c}\right)}\left(t-t_{i}+c\right)^{-p} .
$$

The functions $\mu(t)$ and $K_{0}(t)$ of time are represented by a broken line connecting the respective sequences $\left(t_{i}, \mu\left(t_{i}\right)\right)$ and $\left(t_{i}, K_{0}\left(t_{i}\right)\right)$ for earthquakes $i$, and obtained under the optimal smoothness constraints in piecewise slopes (see Additional file 1: S4 and S5 for more details). Namely, such piecewise functions $\mu(t)$ and $K_{0}(t)$ are characterized by a set of twice many number of coefficient values than those of the earthquakes, which cause 
unstable solutions in maximizing the log-likelihood function with respect to the coefficients of the piecewise functions. To avoid such ill-posed solutions, we consider the penalized log-likelihood (Good and Gaskins 1971):

$$
Q(\theta \mid \tau)=\ln L(\theta, c, \alpha, p)-\text { penalty }\left(\theta \mid w_{\mu}, w_{K_{0}}\right),
$$

where $\theta=\left\{\mu\left(t_{i}\right), K_{0}\left(t_{i}\right)\right\}$ and

$$
\begin{aligned}
\text { penalty }\left(\theta \mid w_{\mu}, w_{K_{0}}\right)= & w_{\mu} \int_{0}^{T}\left\{\frac{\mathrm{d} \mu(t)}{\mathrm{d} t}\right\}^{2} \mathrm{~d} t \\
& +w_{K_{0}} \int_{0}^{T}\left\{\frac{\mathrm{d} K_{0}(t)}{\mathrm{d} t}\right\}^{2} \mathrm{~d} t
\end{aligned}
$$

with regard to hyper-parameters $\tau=\left(w_{\mu}, w_{K_{0}}, c, \alpha, p\right)$.

The objective before obtaining the inversion solution of $\theta$ is to obtain the best selection of hyper-parameter $\hat{\tau}$, which will be listed in Additional file 1: Table S2. Here, the best weights of the penalty for tuning of hyper-parameter values is objectively carried out by the empirical Bayesian procedure using the Akaike Bayesian information criterion (ABIC; Akaike 1980),

$$
\begin{aligned}
\mathrm{ABIC}= & (-2) \max _{\tau}\left\{\log \int \cdots \int L(\theta) \cdot \operatorname{prior}(\theta \mid \tau) \mathrm{d} \theta\right\} \\
& +2 \operatorname{dim}(\tau),
\end{aligned}
$$

where

$$
\operatorname{prior}(\theta \mid \tau)=\exp \{-\operatorname{penalty}(\theta \mid \tau) / 2\} / \int \cdots \int \exp \{-\operatorname{penalty}(\theta \mid \tau) / 2\} \mathrm{d} \theta
$$

is the probability density of $\theta$. Here, we need some major technical cares that are undertaken in a similar manner in Ogata et al. (2003) and Ogata (2011).

We also couple this smoothing procedure with a change-point, which allows a target parameter to have a sharp change or a discontinuous jump at a particular infinitesimal time span, while keeping the variation smoother for the rest of the period. For example, suppose that a large earthquake that occurred at a time $t_{j}$ is suspected to be a change-point of a discontinuity. Then, we add a precursory time point $t_{j}$-near $t_{j}$ to divide the interval $\left(t_{j-1}, t_{j}\right)$ into two subintervals $\left(t_{j-1}, t_{j}-\right)$ and $\left(t_{j}-, t_{j}\right)$, therefore correspondingly dividing the original segment into two segments. The weights for the penalty functions in the precursory interval are set to be the same as those for following intervals, but the weight for the second small interval is a very small value for allowing steep slope of the line, representing a jump. For example, we obtain the values in Additional file 1: Tables S2, S4 and Figs. 3, 5 when we set a sufficiently small constant, $10^{-5}$, in our applications. See Additional file 1: S4 and S5 for more details.

\section{Model comparisons}

We need to compare the goodness-of-fits between the above-mentioned models applied to the same dataset in the entire target interval. We consider the stationary ETAS model (2) as the reference model that is applied to the same dataset in the entire target interval.

First, the two-stage ETAS model is compared based on the increments

$$
\triangle \mathrm{AIC}=\mathrm{AIC}_{12}-\mathrm{AIC}_{0}
$$

Secondly, for the non-stationary ETAS model, we cannot simply compare their resulting ABIC values because of unknown different constants mainly due to the approximations adopted for calculation of (12). Therefore, we use the difference of ABIC values relative to the corresponding to the reference models

$$
\triangle \mathrm{ABIC}=\mathrm{ABIC}-\mathrm{ABIC}_{0},
$$

where $\mathrm{ABIC}_{0}$ is the amount of $\mathrm{ABIC}$ value in the case where the varying coefficients of non-stationary ETAS model are a very heavily penalized, such as $w_{\mu}=\mathrm{w}_{K 0}=10^{5}$. Specifically, $\mu(t)$ and $K_{0}(t)$ become the same as the constant values $\mu$ and $K_{0}$ in of the reference ETAS model, respectively. Such $\mathrm{ABIC}_{0}$ is equivalent to the $\mathrm{AIC}_{0}$ of the reference ETAS model.

It is useful to remember that $\exp \{-0.5 \triangle \mathrm{ABIC}\}$ and $\exp \{-0.5 \Delta \mathrm{AIC}\}$ indicates relative probability of how

likely the non-stationary ETAS model and two-step ETAS model is superior to the reference ETAS model, respectively (Akaike 1980). Furthermore, we compare the non-stationary ETAS model with two-stage ETAS model by comparing $\triangle \mathrm{ABIC}$ and $\triangle \mathrm{AIC}$, via the likelihood $\exp \{-0.5(\triangle \mathrm{ABIC}-\triangle \mathrm{AIC})\}$.

\section{Magnitude frequency analysis in the time and space domains}

The Gutenberg-Richter (GR) law of magnitude frequency is the traditional standard model that has an exponential probability distribution with a completely detected cutoff magnitude, $M_{c}$

$$
\begin{gathered}
F(M \mid b)=1-10^{-b\left(M-M_{c}\right)}=1-e^{-\beta\left(M-M_{c}\right)} \\
M \geq M_{c}, \beta=b \ln 10,
\end{gathered}
$$

where the coefficient is called the $b$-value. In the Hypocenter Catalog of the JMA, the magnitude value is rounded off and given in increments of 0.1. For the purpose of unbiased maximum likelihood estimates (MLEs) and location-dependent spatial estimates of $b$-values, 

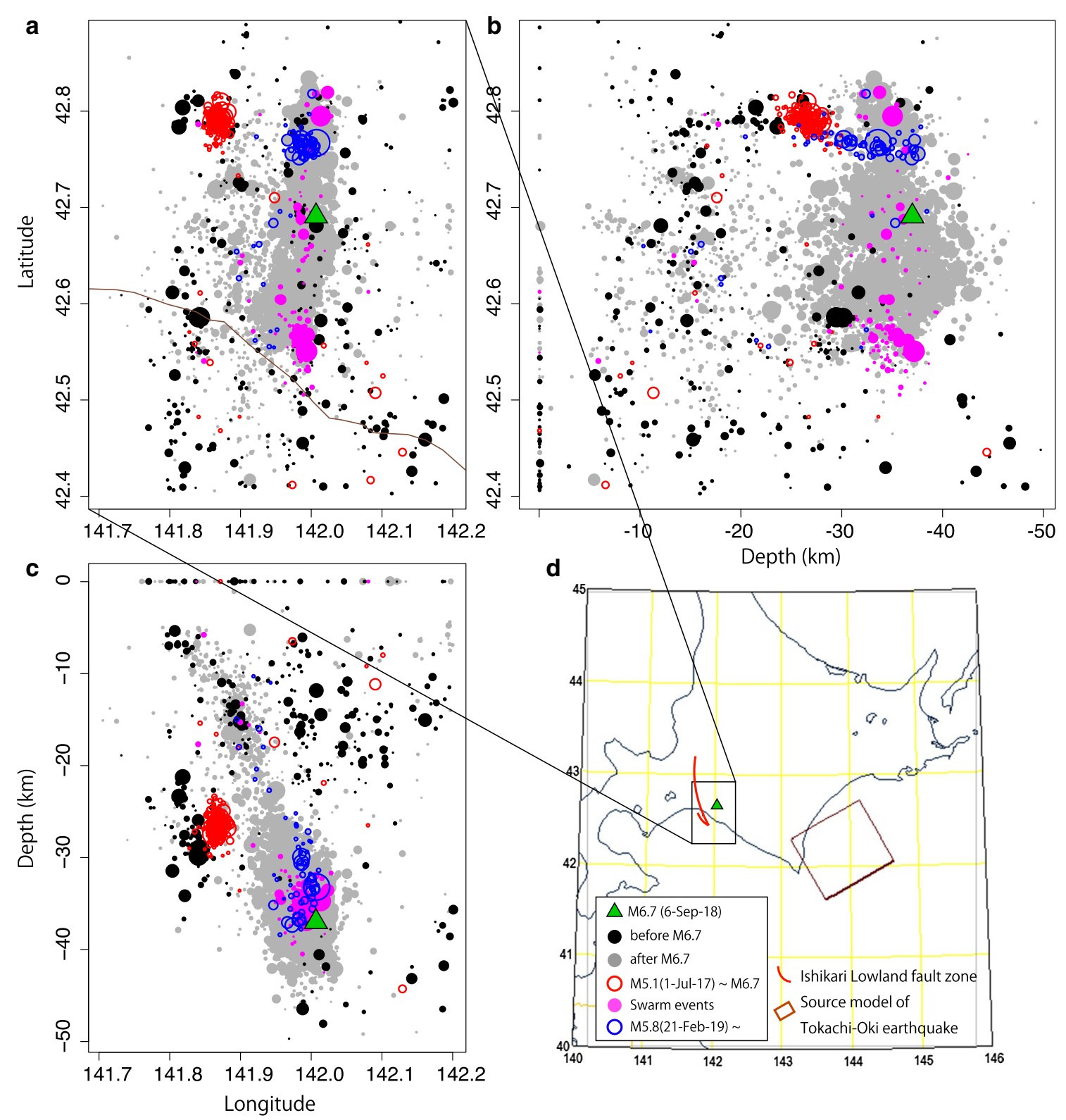

d

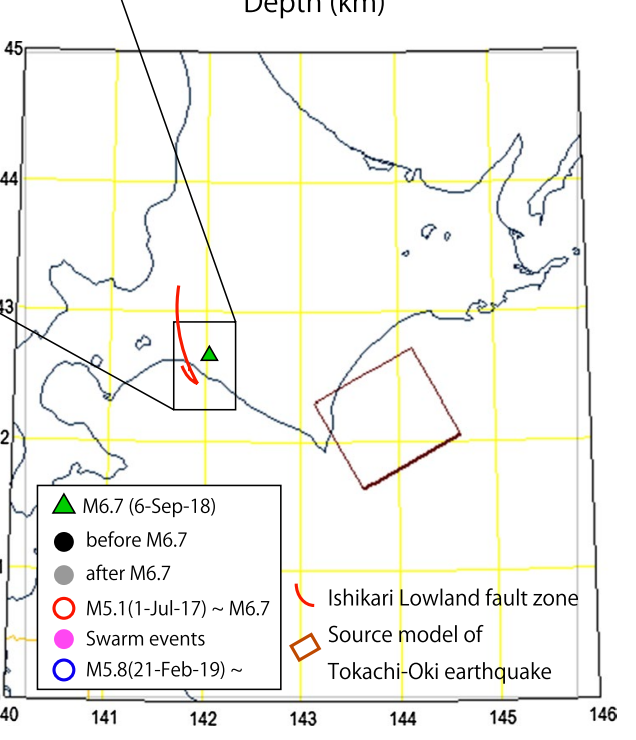

Fig. 1 Hypocenter map in the Eastern Iburi region with $M \geq 1.5$. The three panels show a epicenters, $\mathbf{b}$ longitude vs. depth location, $\mathbf{c}$ depth vs. longitude, and $\mathbf{d}$ Hokkaido map with the Eastern Iburi region and a source model of the M8.0 Tokachi-Oki earthquake (i, 26-Sep-2003) that is listed in Table 1. Gray dots indicate the aftershocks of the M6.7 main shock (iii, 6-Sep-2018) up until March 16, 2019. Black dots indicate the micro-earthquakes before the main shock. Red circles indicate the micro-earthquakes that occurred in between events (ii, 1-Jul-2017) and (iii). Pink circles indicate the micro-earthquakes that occurred 24 days after the main shock (iii), over 4 days. Blue circles indicate the earthquakes after the M5.8 largest aftershock (iv, 21-Feb-2019). The triangle indicates the hypocenter of the main shock (iii). The sizes of disks reflect magnitudes. See Table 1 for the hypocenter coordinates and mechanisms of the cited earthquakes

0.05 should be subtracted from the magnitude of the lower limit (Utsu 1965); for example, we set $M_{c}=1.45$ in (2) for the magnitude data $M \geq 1.5$.

For obtaining the spatial variation of locationdependent $b$-values, we apply the empirical Bayesian smoothing technique assuming a piecewise-linear function $b(x, y)$, using Delaunay tessellation with the locations of earthquakes (Ogata 2011; see Additional file 1: S6). Namely, we use a piecewise-linear function defined by tessellation with triangles (Delaunay 1934; Ogata 2011), with coefficient values for the function given on earthquake locations and additional boundary 


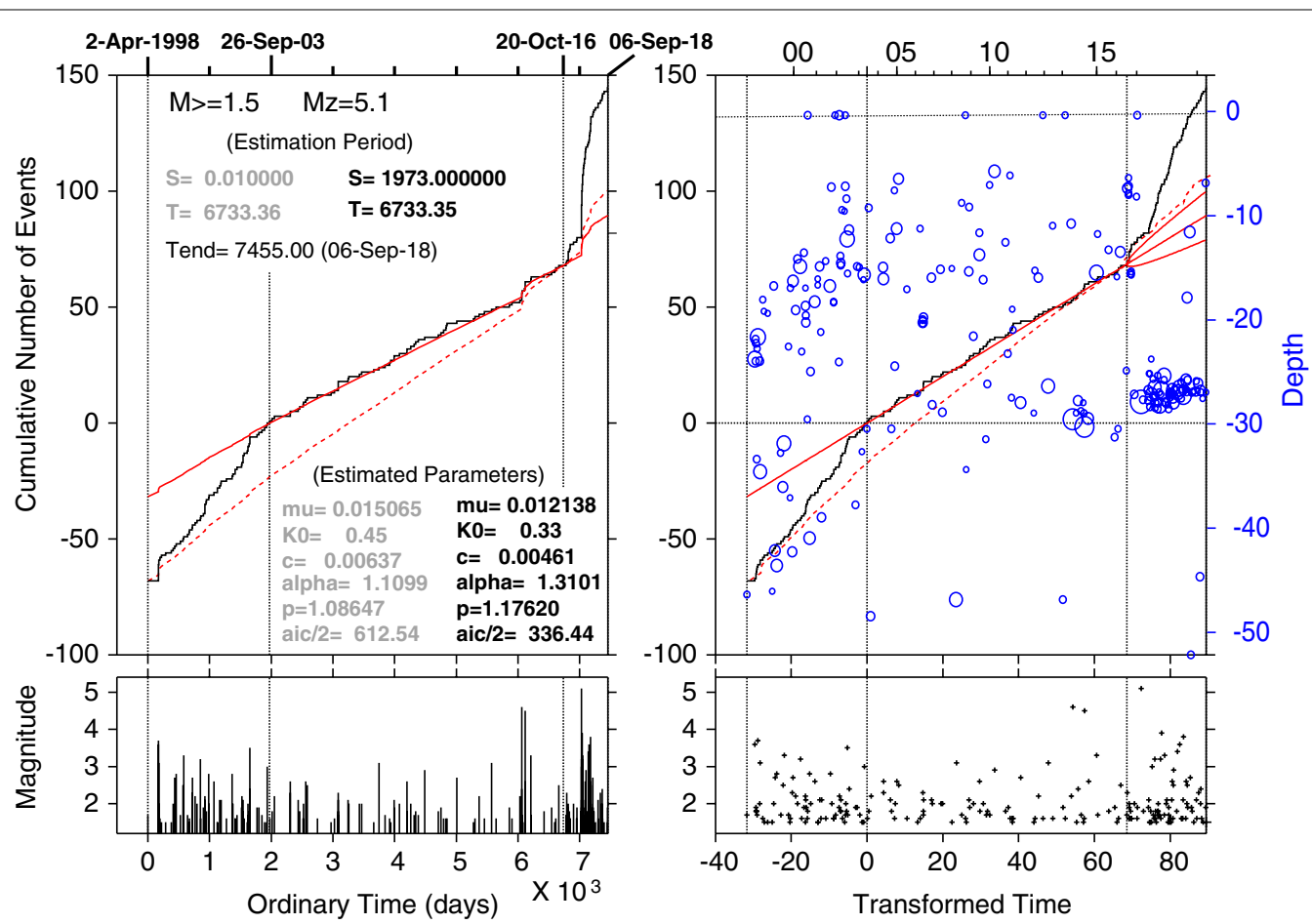

Fig. 2 Two-stage ETAS model fittings during stable period (from 2-Apr-1998 to 20-Oct-2016) before the M6.7 main shock (iii, 6-Sep-2018). Black curves show the observed cumulative number of $M \geq 1.5$ earthquakes from the 2-Apr.-1998. Red curves show the estimated theoretical curve for the target interval from the day of occurrence of the M8.0 Tokachi-Oki earthquake (i, 26-Sep-2003) until the date of the activated seismicity (20-Oct-2016, $t=6733.35$ days from 2-Apr-1998) that led to the M5.1 event (ii, 1-Jul-2017). The estimation period and estimated parameters are printed in black. The left and right plots are in the ordinary time and transformed time (ETAS RPP time), respectively. The parabola shows 2-sigma error bound. The dashed red cumulative curve in each panel shows that of the reference ETAS model fitted for the period from 6-Sep-2018 to 20-Oct-2016. The estimation period and estimated parameters are printed in gray. The blue circles show the depths of the hypocenter against transformed time. Magnitudes against ordinary time (vertical bars) and transformed time (+ signs), regarding the fitted ETAS model, are also plotted. See Additional file 1:Table S2 for the fitting configurations, parameter estimates, and the results of model comparisons

points. Hence, a function value at any location in space is uniquely defined by the linear interpolation of values at the three nearest points (earthquakes) that determine a Delaunay triangle.

We also consider GR law with time-dependent $b$-values for all detected earthquake magnitudes, where the $b$-value function of time is represented by a broken line connecting the sequence of earthquakes under smoothness constraint in piecewise slopes (Kumazawa et al. 2017a, b; see Additional file 1: S7). This method has the advantage of obtaining unbiased $b$-values for time intervals immediately after large earthquakes such as main shocks and large aftershocks, where a substantial number of aftershocks were missed. For estimating such missing rates, the detection rates of earthquakes are modeled using similar broken lines as $b$-values. The optimal weights of the smoothness constraints in variations of the $b$-values and the detection rates are obtained by minimizing the ABIC that is defined by formally same equation as in (6).

\section{Results}

In this work, we analyze datasets obtained from the unified Hypocenter Catalog of the JMA after October 1997 (Fig. 1), in which earthquakes of M1.5 and larger are almost completely detected, except for a short interval immediately after the Iburi earthquake, as shown by magnitude frequency distributions in Additional file 1: Fig. S1.

We retrospectively investigate seismic activity during certain periods before and after the M6.7 main shock, as expressed in the hypocenter colors in Fig. 1.

\section{Seismic activity prior to the Eastern Iburi earthquake}

First, we studied the seismic activity since October 1997 in the region given in Fig. 1 with earthquakes of M1.5 and larger over the entire time period. The conspicuous earthquake that affected the seismicity in this period was the 2003 M8.0 Tokachi-Oki earthquake (i, in Table 1) that occurred about a hundred kilometers in an eastward direction. By comparing the two-stage ETAS model with the reference ETAS model, we see 


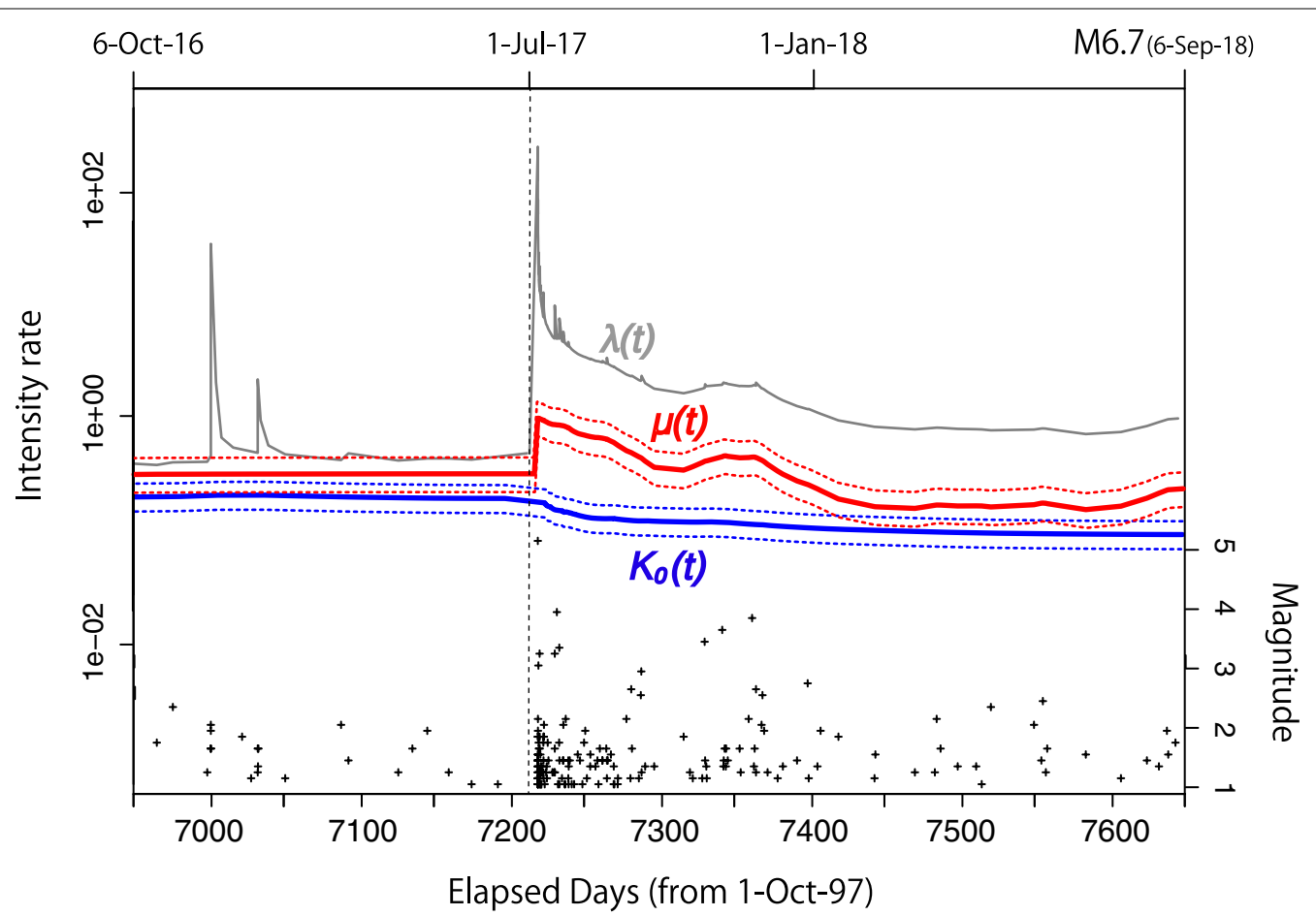

Fig. 3 Non-stationary ETAS estimate right before the M6.7 main shock (iii, 6-Sep-2018). The red and blue curve indicates the background rate $\mu(t)$ and triggering coefficient $K_{0}(t)$ for the period before and after the M5.1 event (ii, 1-Jul-2017), respectively, with 2-sigma error bounds in dashed curves. The spiky gray curve shows the intensity rate $\lambda(t)$ against time. The + signs in the bottom show the magnitudes. See also Additional file 1 : Table $\mathrm{S} 3$ for the fitting configurations and results of the model comparisons

that the micro-seismicity in the focal region became quiet after the Tokachi-Oki earthquake (i, 26-Sep-2003) as shown in Fig. 2 that summarized the AIC difference $(\triangle \mathrm{AIC}=-10.6)$ in Additional file 1: Table S2. Figure 2 also shows the earthquakes were migrated toward the shallower depths until the Tokachi-Oki earthquake occurred.

After earthquake, 14 months before the occurrence of the M6.7 main shock, there was an aftershock cluster that was followed by the M5.1 earthquake (ii) that occurred near the focal zone of the M6.7 earthquake (iii, 6-Sep-2018); see the cluster of red circles in Fig. 1 and the last sub-period in Fig. 2. Therefore, we have applied the non-stationary ETAS model (9) throughout the period from the 1st October 1997 until the M6.7 earthquake (iii). Additional file 1: Table S3 shows that the non-stationary ETAS model is much better than the reference ETAS model, and the model with $\triangle \mathrm{ABIC}=-46.9$ indicating that the change-point for a jump at 1-Jul-2017 is most significant. Figure 3 shows the changes in the background rate $\mu(t)$ and the triggering coefficient $K_{0}(t)$ for the latter part of this period. There is a significant disconnection in smoothing at the time of the M5.1 event (ii, 1-Jul-2017), according to the above-mentioned ABIC comparison (Additional file 1:
Table S2). The background rate $\mu(t)$ and the triggering coefficient $K_{0}(t)$ were constant until the M5.1 event (ii) occurred, at which time the background rate $\mu(t)$ jumped nearly tenfold and then decreased gradually to lower than the previous level. After this the background rate recovered slightly before the M6.7 main shock (iii). The triggering coefficient $K_{0}(t)$ stayed almost constant throughout.

\section{Aftershock activity following the M6.7 main shock}

On September 6, 2018, a M6.7 earthquake (iii, 6-Sep2018) occurred at a depth of $37 \mathrm{~km}$; subsequently, on February 21, 2019, the M5.8 largest aftershock (iv, 21-Feb-2019) occurred at a depth of about $35 \mathrm{~km}$ in the northern part of the primary aftershock zone of the M6.7 main shock (see Table 1 and Fig. 1).

\section{Seismicity characteristics of the entire aftershock volume}

The aftershocks of the M6.7 earthquake up to the end of the period (March 16, 2019), with the cut-off magnitude $M_{c}=1.5$, were analyzed by fitting to the stationary ETAS model in (2). Since the aftershocks immediately after the main shock are incompletely detected, we set the first 0.1-day interval is precursory period in (4); and 

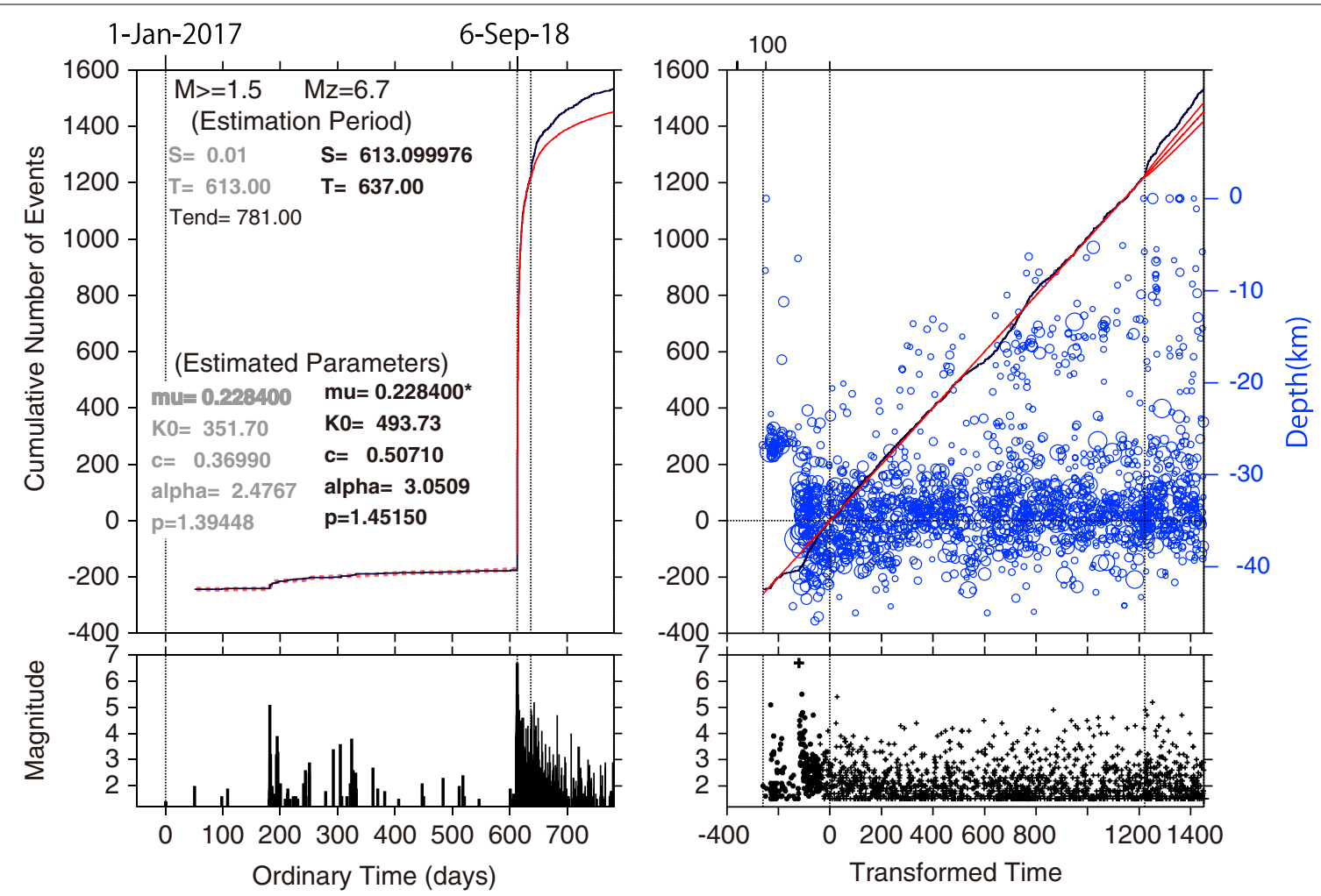

Fig. 4 Aftershock activity $(M \geq 1.5)$ of the main shock (iii, 6-Sep-2018) and the ETAS estimate. The ETAS model is fitted during the target interval from 0.1 day after the M6.7 main shock (iii) (2nd vertical dashed line from the left) until 23.4 elapsed days (1-Oct-2018; 3rd vertical dashed line). The estimation period and estimated parameters are printed in black. The background rate of the aftershock activity is fixed $(\mu=0.228)$. This value is the MLE of the ETAS $\mu$ in the interval before the M6.7 earthquake (iii); this fit is plotted by thick dashed red curve, with the estimation period and estimated parameters printed in gray. Theoretical cumulative curves were then extrapolated to the last period. The empirical cumulative curve (black) is superimposed by estimated and extrapolated curve (red). The blue circles show the depths of the hypocenter against transformed time. See Additional file 1:Table S4 for the fitting configurations and results of model comparisons

we get the MLEs from the rest as the target period, where the background rate of the aftershock activity is fixed $(\mu=0.228$ event per day) that is the MLE of the ETAS $\mu$ in the preceding interval before the M6.7 earthquake (iii, 6-Sep-2018). Also, the background rate $\mu=0.228$ is within the error bounds of the background rate of the preceding non-stationary ETAS model (Fig. 3).

Additional file 1: Table S4 (whole period) shows that the occurrence time of (iv, 21-Feb-2019), the M5.8 largest aftershock, is a clear change-point with $\triangle \mathrm{AIC}=-47.9$, which means that the ETASmodel of the primary aftershock activity following the main shock has different parameters from those of the secondary aftershock activity that followed the M5.8 event (iv). In particular, the ETAS triggering coefficient $K_{0}$ of the M5.8 event (iv) is significantly lower than that of the M6.7 main shock (iii).

There is another change-point in the period before the occurrence time of the M5.8 event (iv) (see the top half rows of Additional file 1: Table S4). We first analyzed the aftershock period between the events (iii) and (iv), and found a significant change-point with $\triangle \mathrm{AIC}=-21.2$ on the 24th day (1-Oct-2018) after the main shock (iii). Figure 4 shows that cumulative curve of the seismicity obeys that of the stationary ETAS model (red curve) until the time of the change-point, but then it deviates upwards for several days, and the RPP cumulative curve deviates outside the 2-sigma parabola; in other words, it becomes relatively active compared to the ETAS prediction. It then recovers later. Furthermore, the RPP plot of blue circles in Fig. 4 shows seeming migration of aftershocks' depth distribution, which is related to the increase in percentage of shallow aftershocks greater than about $25 \mathrm{~km}$ depth relative to the decaying trend of the aftershock activity in the deeper part.

We further applied the non-stationary ETAS model (9) throughout the period from the M6.7 main shock (iii) until the end of the period (16-Mar-2019), with the same precursory period of 0.1 days after the M6.7 event (iii) as the previous two-stage ETAS model, in consideration of lower detection rate. Note there is little discrepancy in 


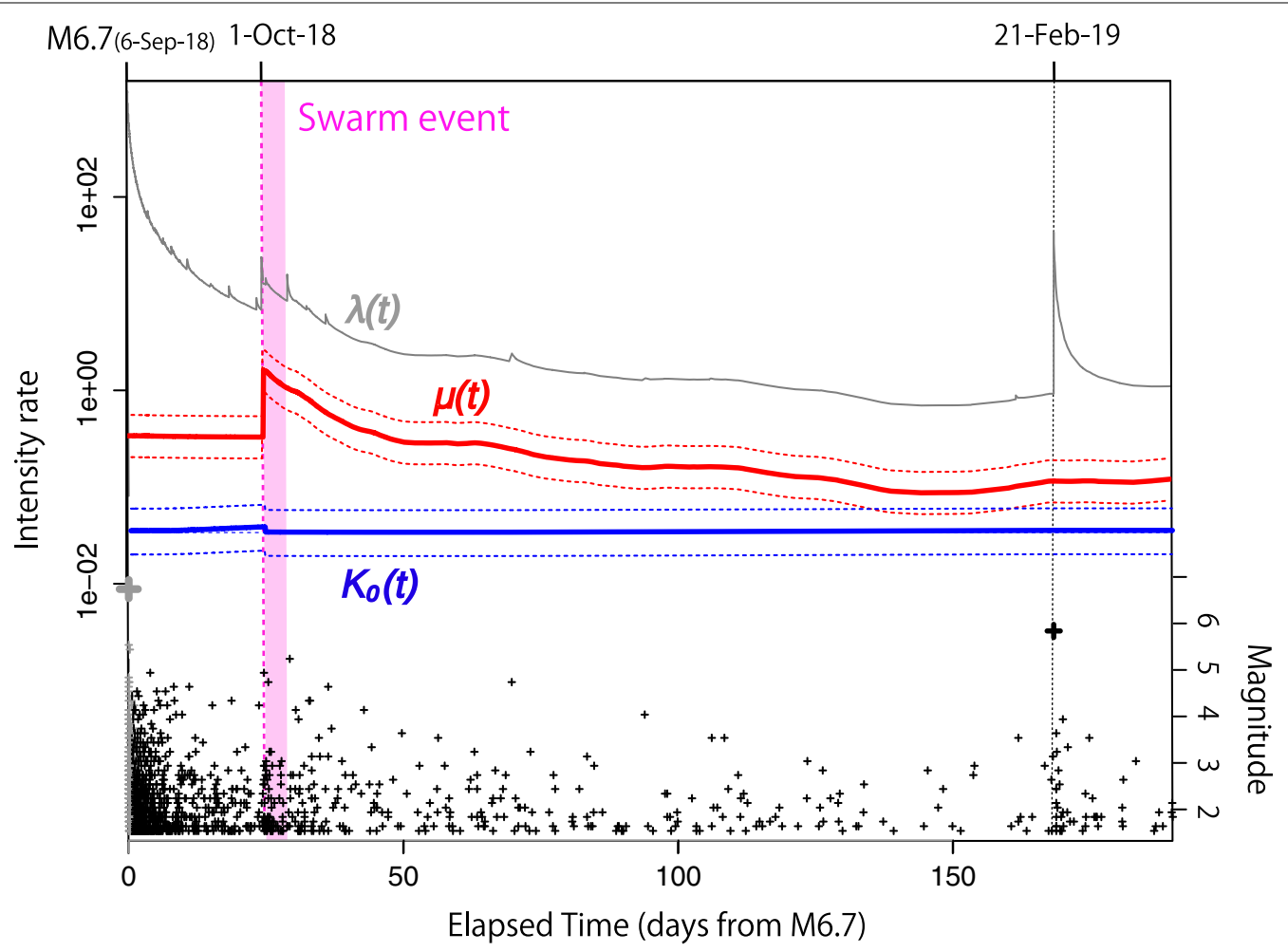

Fig. 5 Estimated non-stationary ETAS model, where the MLEs of the ETAS model, as shown in Fig. 4, were used as the reference parameters (see Additional file 1: S4). The background rate $\mu(t)$ (red) and the triggering coefficient $K_{0}(t)$ (blue) in the ordinary time; the envelopes of the twofold standard errors of the estimations are indicated by dotted lines. The gray curve shows the whole intensity $\lambda(t)$. The first vertical dashed line shows the change-point (1-Oct-2018) as also shown in Fig. 4, and the pink shade indicates the span of the following 4 days. The second vertical dashed line is at the occurrence of the M5.8 largest aftershock (iv). The thick + signs indicate magnitude of the main shock (iii) and the largest aftershock (iv), against occurrence times. See Additional file 1: Table S5 for the fitting configurations and results of model comparisons

$\mu(t)$ before and after the M6.7 event (iii) according to the last column of Additional file 1: Tables S3, S5 and Figs. 3 and 5; namely, the estimated value of $\mu(t)=0.215$ with $95 \%$ credible interval $(0.175-0.255)$ for the former, and 0.233 with $(0.198,0.268)$ for the latter).

Figure 5 shows the changes in the background rate $\mu(t)$ and the triggering coefficient $K_{0}(t)$. There is a significant disconnection in smoothing at the time of the changepoint (1-Oct-2018) with, whereas no discontinuity at the time of the M5.8 largest aftershock (iv, 21-Feb-2019), according to the ABIC comparison (Additional file 1: Table S5). At the change-point, the background rate $\mu(t)$ increased nearly tenfold, and then decreased gradually until it was lower than the level before the changepoint. Thereafter the rate recovered slightly before the M5.8 event (iv), whereas the triggering coefficient $K_{0}(t)$ remained almost constant. It can be seen from Fig. 1 that the seismic activity for 4 days after the change-point (pink-colored band in Fig. 5) is spatially concentrated at southern end of the aftershock volume in purple color disks, where we suspect that might be the swarms caused by water intrusion as discussed later.
On the other hand, Fig. 6 summarizes the analyzed results in Additional file 1: Table S6 for the aftershock activity with magnitude thresholds of $M_{c}=3.0$ and 4.0, where the change-point hypothesis is supported by the $\triangle \mathrm{AIC}$ value -6.9 and -2.2 , respectively; and significant quiescence is seen from their RPP cumulative curves deviating outside the 2-sigma parabola. These are larger thresholds than the previous cases with a micro-seismicity of $M_{c} 1.5$. Even with these moderate-sized aftershocks, significant relative quiescence is found from 6 days after the M6.7 main shock (iii), and it lasts until the M5.8 largest aftershock (iv) by the ETAS model.

\section{Further seismicity characteristics for divided zones of the Eastern Iburi aftershocks}

Because the aftershocks of the M6.7 main shock (iii, 6-Sep-2018) with the low cut-off magnitude $\left(M_{c}=1.5\right)$ are abundant, we studied the whole aftershock sequence in more detail by dividing the entire aftershock area into four spatial zones as follows. We first divided shallow and deep zone by visually consulting the clustering of earthquakes at the depth of $23 \mathrm{~km}$ by looking at the 

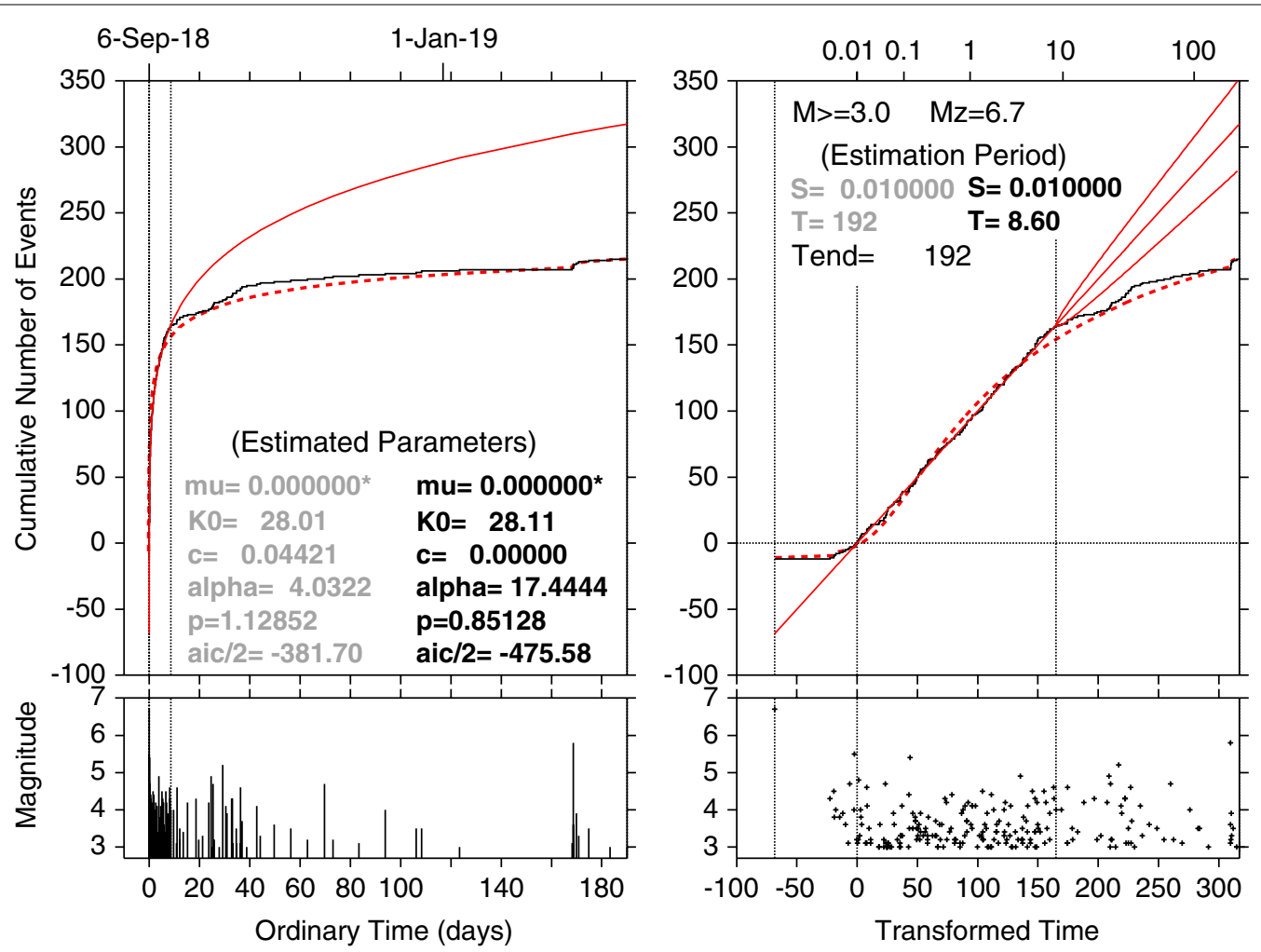

Fig. 6 ETAS estimate for aftershock activity $(M \geq 3.0)$ to the target period from the main shock (iii) until 8.6 days had elapsed (14th Sept. 2018) (with the estimation period and estimated parameters printed in black), and then extrapolated to the rest of the period. The ordinary time plot is on the left, transformed time plot is on the right. The empirical cumulative (black) curve deviated downwards outside the twofold error parabola from the expected trend, showing relative quiescence after the change-point. The dashed cumulative curves show those of the reference ETAS model for the entire period (with the estimation period and estimated parameters in gray). See Additional file 1: Table S6 for the fitting configurations and results of model comparisons

distributions of gray disks in Fig. 1b and c, Fig. 9a and b, and also the different activity patterns seen in RPP depths against transformed times in Fig. 4; we further subdivided the deep zone into northern, middle, and southern zones, as shown in Fig. 7a. Here, for the calculation of the MLE and AIC,we include the M6.7 main shock (iii) occurrence data in the log-likelihood function (3) for every zone because the main shock magnitude is too large for such a narrow region to ignore the triggering effect.

The deep middle zone that actually includes the main shock obeys the stationary ETAS model as seen in Fig. 7c based on the results in Additional file 1: Table S7 (center). However, the northern and southern zone shows opposite anomaly of relative quiescence and activation, according to Fig. $7 \mathrm{~b}$ and $\mathrm{d}$ whose $\triangle \mathrm{AIC}$ value is -12.5 and -11.6 in Additional file 1: Table S7 (North) and (South), respectively. Their cumulative curves deviate outside the 2-sigma parabolas. It is noteworthy that both change-points are the same.

For the activity in the shallow zone, none of the stationary and two-stage ETAS models appears to provide satisfactory fits according to Fig. 8a and b, respectively. Therefore, we applied the non-stationary ETAS model (4), which shows a significantly better fit, according to the $\triangle \mathrm{ABIC}$ in Additional file 1: Table S8 and thick gray cumulative curve in Fig. 8a. The estimated background rate $\mu(t)$ and triggering coefficient $K_{0}(t)$ with the cumulative function for shallow activity are given in Fig. $8 \mathrm{c}$ in comparison with those of the stationary ETAS model.

\section{Characteristic changes in $b$-values of the GR magnitude distribution}

The time change of the $b$-values (black curve in Fig. 9c) of the aftershocks, taking account of the time dependence of earthquake detection rates (Additional file 1: S7), was obtained using all the detected data throughout the period from the main shock (iii, 6-Sep-2018) up to the end of the period (16-Mar-2019). Some undulation can be observed in the $b$-values with respect to the lapse time plotted in logarithmic scale, but a gentle increasing trend is recognizable in the black curves. 


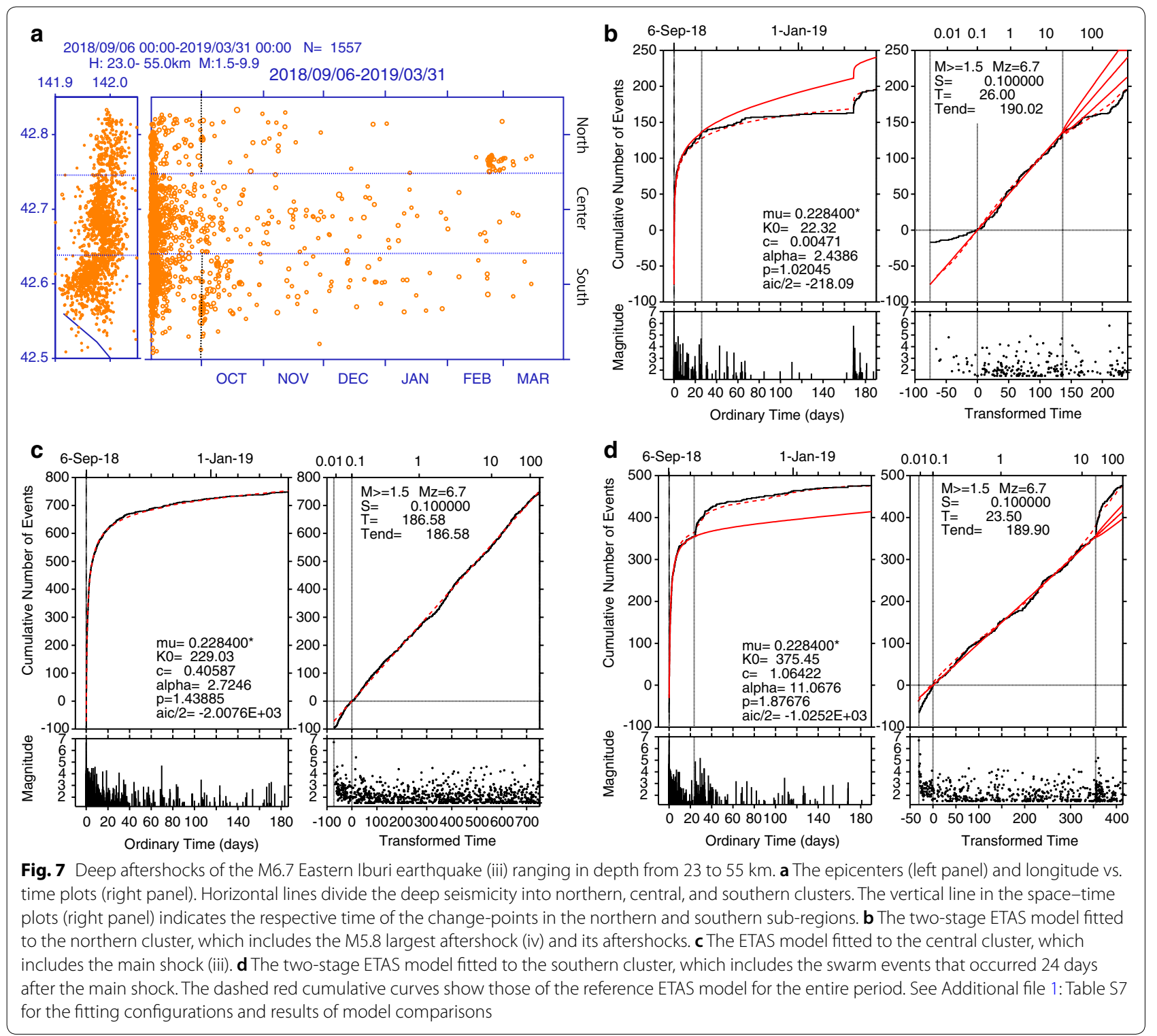

However, the two-dimensional spatial distribution projected onto the tomographic plane of $b$-values have clear location-dependent variation (see Additional file 1: S6), especially where the shallow $b$-values are larger than 0.85 , whereas deeper $b$-values show patchy patterns but much smaller than 0.85 (Fig. 10). These patterns were objectively obtained according to the optimal solution of the ABIC procedure (Additional file 1: Table S10). The patchiness in the deeper area resulted from the far denser earthquakes. For smoothing the b-value, we use a piecewise-linear function defined on the Delaunay tessellated region. The vertices of each Delaunay triangle comprise the hypocenter coordinates of the three nearest earthquakes, hence the resolution is finer with more hypocenters.
Therefore, we divided the aftershock zone into shallow and deep sections; according to the solution of the ABIC analysis in Additional file 1: Table S10, the $b$-value time changes for the shallow part are chosen to be a constant due to the very high weight $w 1$. However, the $b$-value for the deep section shows a smaller increasing trend than that for the aftershocks in general (Fig. 9c).

The ratio of the numbers of the deep aftershocks relative to the shallow aftershocks in Fig. 9e appears to relate the undulation of the $b$-values of the entire aftershocks. See also Fig. 4 for the migration and relative increase of shallow aftershocks relative to the deep ones. Consequently, we imply that the increasing trend and undulation of $b$-values of the entire aftershocks in Fig. $8 \mathrm{c}$ is due 

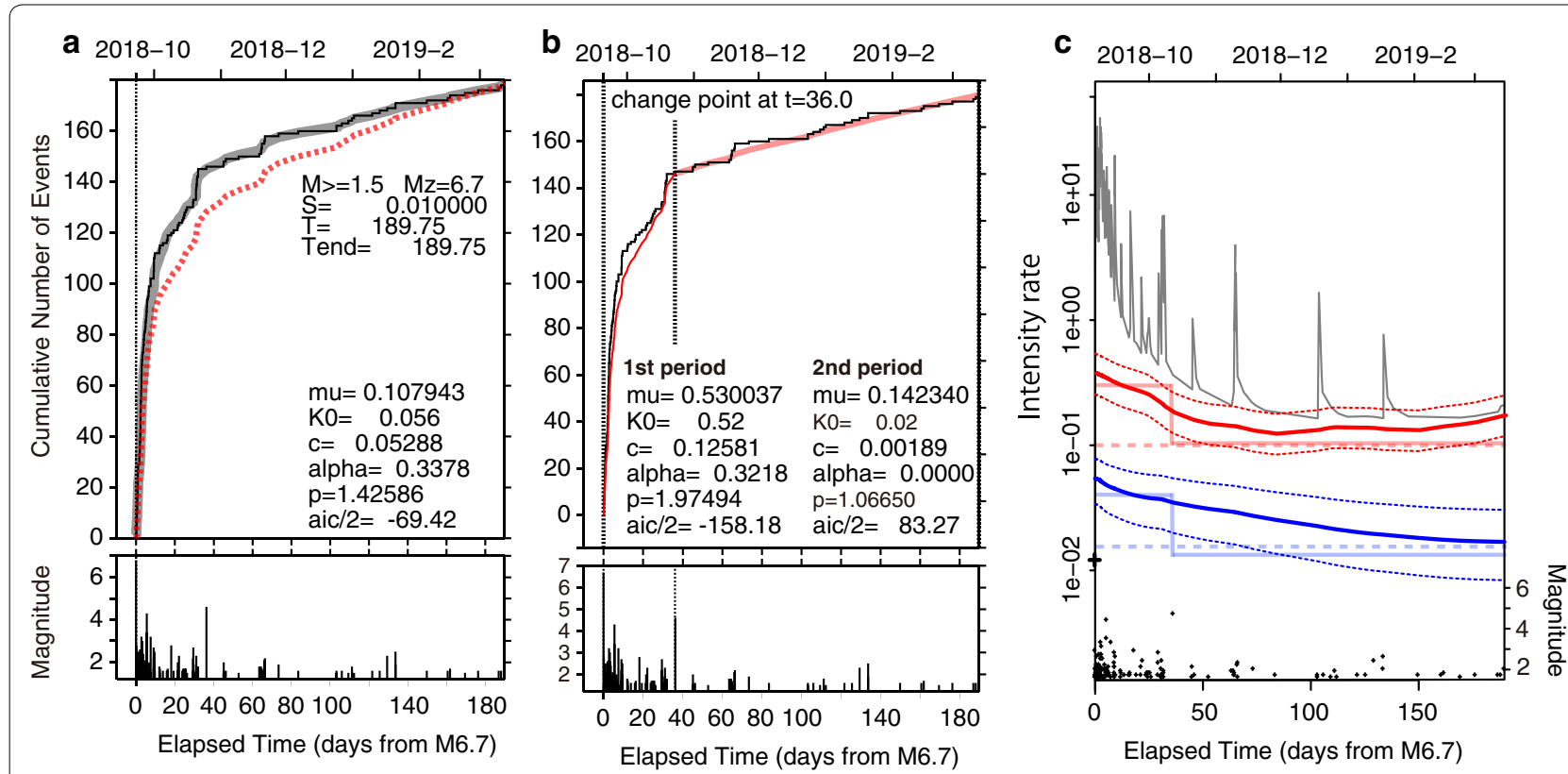

Fig. 8 Estimates of stationary ETAS model, two-stage ETAS model and non-stationary ETAS model. The models were applied to a seismicity of $M \geq 1.5$ in the shallow depth range down to $23 \mathrm{~km}$. a The black curve shows the empirical cumulative number of the shallow aftershocks; the red dashed curve shows the theoretical cumulative estimate of the ETAS model. Beneath the empirical cumulative curves, the thick light gray curve indicates the theoretical cumulative curves of the non-stationary ETAS model. The dashed red cumulative curves show those of the reference ETAS model for the entire period. $\mathbf{b}$ The two-stage ETAS model is fitted to the periods separated by the change-point at 36 days. The parameter estimates are listed. The dashed red cumulative curves show those of the two-stage ETAS model for respective period. c The non-stationary ETAS model estimate: $\mu(t)(\mathrm{red})$ and $K_{0}(t)$ (blue) with twofold errors. Pale solid and dashed horizontal lines, respectively, indicate background rate (red) and triggering coefficient (blue) of the two-stage ETAS and the reference model. The spiky gray line is the theoretical occurrence rate of the non-stationary ETAS model, and the + signs indicate magnitude vs. time of the shallow aftershocks. See Additional file 1: Table S8 for the fitting configurations and results of model comparisons

to the ratio of the occurrence of shallow aftershocks relative to the deep ones (Fig. 9e).

\section{Discussion and conclusions}

Seismic activity in and surrounding the area of the aftershock zone of the 2018 M6.7 Eastern Iburi earthquake (iii, 6-Sep-2018) was analyzed using the ETAS model, the non-stationary ETAS model (4) and the model of the magnitude frequency including seismic detection rate.

The triggering effects of the M8.0 Tokachi-Oki earthquake (i, 26-Sep-2003) (see the source model in Fig. 1, Geographical Survey Institute 2003) on the seismicity in the focal region are complex. Firstly, occurrences of the 2018 M6.7 Eastern Iburi earthquake (iii) and the preceding neighboring moderate M5.1 earthquake (ii, 1-Jul-2017) seems to be promoted by the Coulomb failure stress increases of about +1.4 bars and +0.3 bars, respectively; here we used the receiver fault mechanisms listed in Table 1 and source fault parameters given by the Geographical Survey Institute (2018) and the F-net catalog of National Research Institute for Earth Science and Disaster Resilience (2019). On the other hand, the micro-seismicity in the focal region became quiet after the Tokachi-Oki earthquake (i) as seen in Fig. 2. This might suggest that the entire region is covered by stress shadow as discussed by Ogata (2006a, b, 2007, 2011).

The micro-aftershock activities of the both M5.1 (ii) and M6.7 (iii) earthquakes, with M1.5 and larger, show transient increases in background rates, as seen in Figs. 3 and 5. These suggest that swarm activities may have been induced by fluids weakening local faults. Such behavior was observed during swarms in Vogtland/NW Bohemia, central Europe (Hainzl and Ogata 2005), during several unusual earthquake events triggered by the 2011 TohokuOki earthquake of M9.0 in northern Japan (Terakawa et al. 2013; Kumazawa and Ogata 2013), in particular near the Lake Inawashiro (Kumazawa and Ogata 2013; Yoshida et al. 2017), and in Basel enhanced geothermal system, Switzerland (Terakawa 2014).

The moderate magnitude sized aftershock activity of the M6.7 event (iii) became significantly quiet relative to the occurrence rates in the ETAS model fitted for the first period after the main shock (iii). For example, applying the ETAS model to the aftershock activity with the 


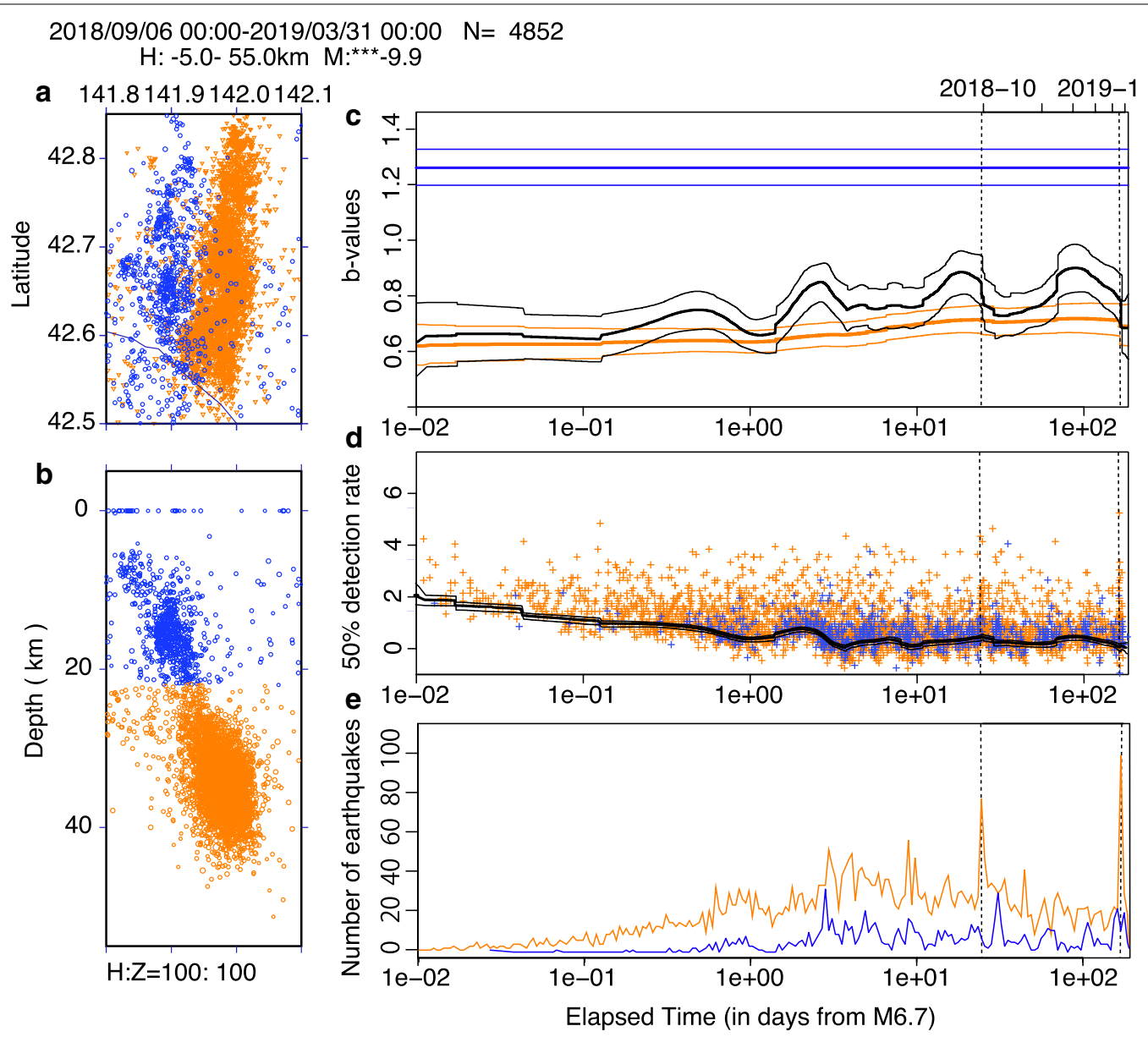

Fig. 9 Time-dependent $b$-value estimates and the $50 \%$ detection rate of magnitudes. a Epicenters of the whole aftershocks of $M \geq 1.5$; orange and blue dots indicate the deep aftershocks at 23-55 km depth and the shallow aftershocks at 0-23 km depth, respectively. $\mathbf{b}$ Hypocenters are plotted as depth vs. longitude. c Time-dependent $b$-values are estimated with the aftershocks at all depths (black line), shallow aftershocks (blue line), and deep aftershocks (orange line), respectively; these are with twofold error envelopes. $\mathbf{d}$ The + signs show $M-T$ plots with colors for corresponding ranges in depth. The black line indicates a $50 \%$ detection rate of magnitudes for all depths with twofold errors together with magnitudes of all detected aftershocks. $\mathbf{e}$ The blue and orange broken line shows counted numbers of the shallow and deep aftershocks, respectively, counted in bins of 0.05 logarithmic days from the M6.7 main shock. The dashed vertical lines throughout c-e indicate the occurrence of the topical swarm events and the M5.8 largest aftershock (iv), respectively. See Additional file 1: Table S9 for the fitting configurations and results of model comparisons

lower limits of $M_{c}=3.0$ and 4.0 suggested that the region became relatively quiet about 1 week after the main shock (iii), and then recovered during a month; but then lowered again until the occurrence of the M5.8 largest aftershock (iv, 21-Feb-19) (see Fig. 6).

We speculate one possible scenario as a reason for the earlier part of the quiescence (Kumazawa et al. 2018; see also Additional file 1: S8). It was suggested that the main shock dynamically weakened a part of the shallow faults in the eastern margin of the Ishikari Lowland fault zone, causing a "slow slip", which caused the aftershock area of the Eastern Iburi earthquake to become a stress shadow. After that, the strength of the fault zone may have recovered, but the weakened stress shadow might have triggered the sequence of strong aftershocks that followed.

Furthermore, the relative quiescence that lasted until the M5.8 largest aftershock (iv) may be explained by the stress shadow by the precursory slow slip on the M5.8 fault and its extension. Although the relative quiescence cannot be seen for the entire aftershock volume with the cut-off magnitude $M_{c}=1.5$ (Fig. 4), we see the relative quiescence in the northern zone of the deep part of the aftershock volume that includes the M5.8 earthquake (iv). The other three parts of the M6.8 Iburi earthquake show contrastingly different patterns to each other. Readers are 

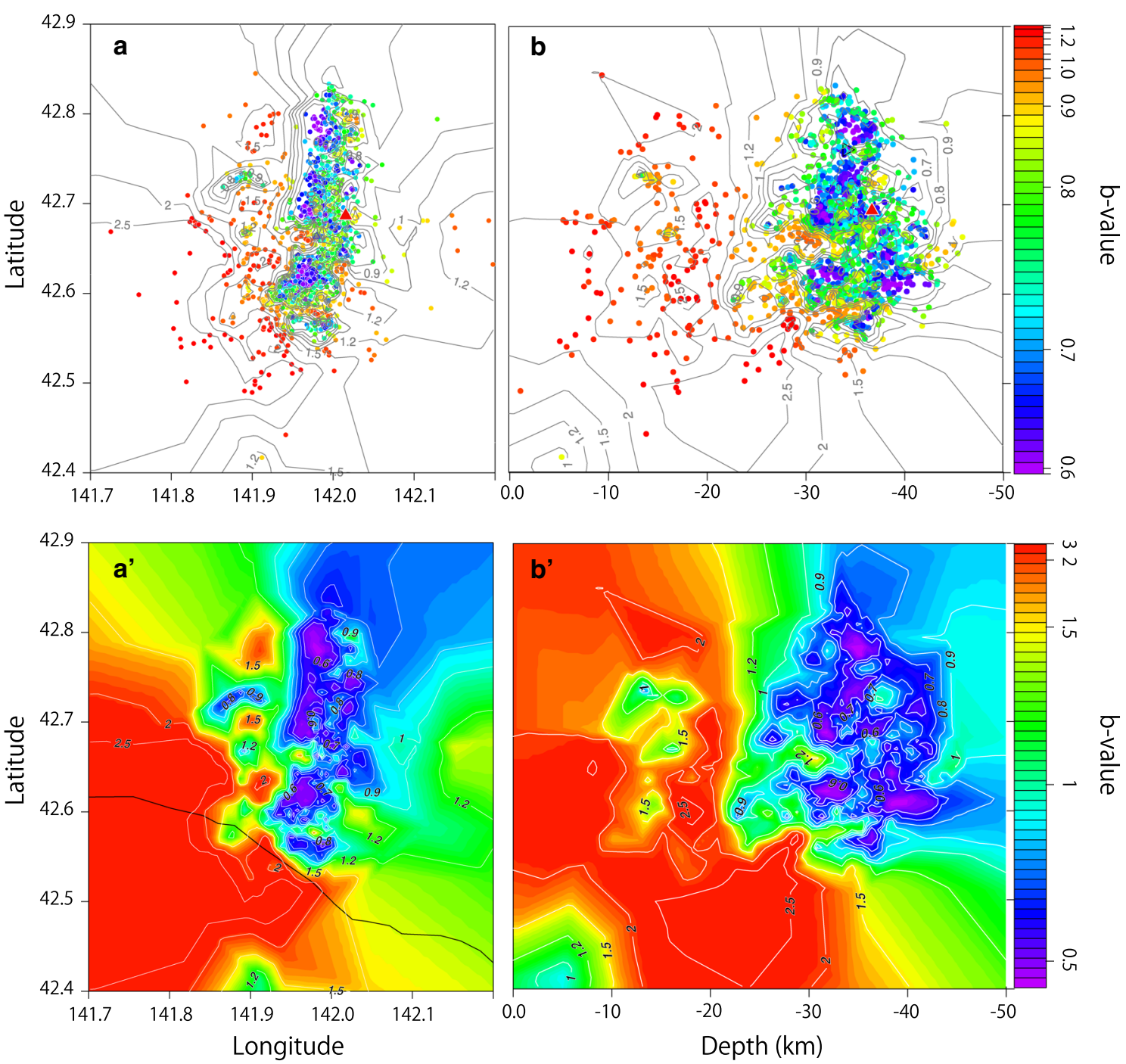

Fig. 10 Spatial $b$-value estimates of the entire aftershocks of $M \geq 1.5$ of the $M 6.7$ main shock. The upper panel $(\mathbf{a}, \mathbf{b})$ shows the colored estimated $b$-values on the hypocenters together with contours of interpolated image, projected onto the horizontal and the vertical plane, respectively. The lower panels only show the interpolated coloring of the $b$-values with contours, but with different color ranges, so as to highlight the differences in values between deep and shallow earthquakes. The red triangle indicates the main shock (iii). See Additional file 1: Table S10 for the fitting configurations and results of modelcomparisons

also referred to Katsumata (2019) for mechanism about the three separations.

The $b$-values of the entire aftershock region seemingly increased in trend with some undulation during the period after the main shock (iii), which is explained by the spatially different $b$-values and different fluctuating occurrence rates of the shallow and deep aftershocks.

\section{Supplementary information}

Supplementary information accompanies this paper at https://doi. org/10.1186/s40623-019-1102-y.
Additional file 1. The sections S1-S5 provides detailed explanations of the ETAS model and its variations. The section $\mathrm{S} 6$ describes changes in $b$-values of magnitude frequency distribution in space and time. The section S7 (with Fig. S1) explains earthquake detection rates in time. The section S8 (with Fig. S2 and Table S1) describes a scenario of seismicity quiescence of the aftershock sequence before the M5.8 largest event. The section S9 lists the tables (Table S2-Table S10) for the model comparisons.

\section{Abbreviations}

ABIC: Akaike Bayesian information criterion; AIC: Akaike information criterion; ETAS model: epidemic-type aftershock sequence model; MLE: maximumlikelihood estimate; XETAS: X-windows-based ETAS applications. 


\section{Acknowledgements}

We used the PDE Hypocenter Earthquake Catalog (as of March 2019) of the JMA. We would like to thank to the JMA, the National Research Institute for Earth Science and Disaster Resilience (NIED), and the relevant universities for providing hypocenter data, and to the Meteorological Laboratory for MICAP-G that calculates Coulomb failure stress. We are grateful for the support from the Japan Society for the Promotion of Science KAKENHI Grants 16 K00065 and 17H00727. We also thank the support of the Tokyo Metropolitan Resilience Project.

\section{Authors' contributions}

TK arranged the statistical tools, modified and extended the functions used for the current study for implementing the analysis, made figures and tables, and also wrote the manuscript. YO organized the study, drafted the original manuscript, and had prepared some original statistical programs for the methods. HT had made and modified the XETAS programs for the present study. YO and $\mathrm{HT}$ checked the results. All authors read and approved the final manuscript.

\section{Funding}

This research is funded from the Japan Society for the Promotion of Science KAKENHI Grants 16K00065 and 17H00727, and the Tokyo Metropolitan Resilience Project.

\section{Availability of data and materials}

The JMA hypocenter earthquake catalog can be downloaded from the JMA home page (https://www.jma.go.jp/jma/index.html). The F-net catalog can be found in the NIED home page (http://www.bosai.go.jp/).

\section{Ethics approval and consent to participate}

Not applicable.

\section{Consent for publication}

Not applicable.

\section{Competing interests}

The authors declare that they have no competing interests.

\section{Author details}

${ }^{1}$ Earthquake Research Institute, University of Tokyo, Tokyo, Japan. ${ }^{2}$ The Institute of Statistical Mathematics, Tachikawa, Japan.

Received: 25 April 2019 Accepted: 29 October 2019

Published online: 29 November 2019

\section{References}

Akaike H (1973) Information theory and an extension of the maximum likelihood principle. In: Petrov BN, Csaki F (eds) 2nd international symposium on information theory. Akademiai Kiado, Budapest, pp 267-281

Akaike H (1974) A new look at the statistical model identification. IEEE Trans AC $19: 716-723$

Akaike H (1977) On entropy miximisation principle. In: Proceedings of symposium on applications of statistics, pp 27-47

Akaike H (1980) Likelihood and Bayes procedure. In: Bernard JM, De Groot MH, Lindley DU, Smith AFM (eds) Bayesisn statistics. University Press, Valencia

Bansal AR, Ogata Y (2013) A non-stationary epidemic type aftershock sequence model for seismicity prior to the December 26, 2004 M9.1 Sumatra-Andaman Islands mega-earthquake. J Geophys Res Solid Earth 118:616-629

Delaunay B (1934) Sur la sphère vide. Bull Acad Sci URSS 6:793-800

Earthquake Research Committee (2010) About the partial revision of longterm evaluation of the eastern margin of the Ishikari Lowland Fault Zone, August 26. https://www.jishin.go.jp/evaluation/long_term_evaluation/ major_active_fault. Accesed 10 July 2019

Geographical Survey Institute (2003) Crustal Movements in the Hokkaido District, Report of the Coordinating Committee of Earthquake Prediction, Japan. https://www.jishin.go.jp/main/index-e.html, https://cais.gsi.go.jp/ YOCHIREN/report/kaihou71/01-14.pdf. Accessed 10 July 2019
Geographical Survey Institute (2018) Seismic Fault Model of the 2018 Hokkaido Ballistic Eastern Earthquake (provisional) Earthquake Research Committee, October 12, 2018. https://www.static.jishin.go.jp/resource/ seismicity_annual/major_act/2018/20180906_iburi_10_sourcemode l.pdf. Accessed 10 July 2019

Good IJ, Gaskins RA (1971) Nonparametric roughness penalties for probability densities. Biometrika 58:255-77

Hainzl S, Ogata Y (2005) Detecting fluid signals in seismicity data through statistical earthquake modeling. J Geophys Res 110:B05S07. https://doi. org/10.1029/2004JB003247

Katsumata K et al (2019) The 2018 Hokkaido Eastern Iburi earthquake (MJMA =6.7) was triggered by a strike-slip faulting in a stepover segment: insights from the aftershock distribution and the focal mechanism solution of the main shock. Earth Planets Space 71:53. https://doi. org/10.1186/s40623-019-1032-8

Kobayashi T, Hayashi K, Yarai H (2019) Geodetically estimated location and geometry of the fault plane involved in the 2018 Hokkaido Eastern Iburi earthquake. Earth Planets Space 71:62. https://doi.org/10.1186/s4062 3-019-1042-6

Kumazawa T, Ogata Y (2013) Quantitative description of induced seismic activity before and after the 2011 Tohoku-Oki Earthquake by non-stationary ETAS models. J Geophys Res 118(6165-6182):1

Kumazawa T, Ogata Y, Toda S (2010) Precursory seismic anomalies and transient crustal deformation prior to the $2008 \mathrm{Mw}=6.9$ Iwate-Miyagi Nairiku, Japan, earthquake. J Geophys Res 115:B10312

Kumazawa T, Ogata Y, Kimurac K, Maeda K, Kobayashi A (2017a) Background rates of swarm earthquakes that are synchronized with volumetric strain changes. Earth Planet Sci Lett 442:51-60

Kumazawa T, Ogata Y, Tsuruoka H (2017b) Measuring seismicity diversity and anomalies by point process models: case studies before and after the 2016 Kumamoto Earthquakes in Kyushu, Japan. Earth Planets Space 69:169. https://doi.org/10.1186/s40623-017-0756-6 (Open Access)

Kumazawa T, Ogata Y, Tsuruoka H (2018) Characteristics of aftershock activity of the Hokkaido Eastern Iburi Earthquake, to appear in Rep Coord Commun Earthq Predic Japan. The Institute of Statistical Mathematics, p 32. https://cais.gsi.go.jp/YOCHIREN/activity/221/221.html, https://cais.gsi. go.jp/YOCHIREN/activity/221/221.e.html. Accessed 10 Apr 2019

National Research Institute for Earth Science and Disaster Resilience (NIED) (2019) F-net broadband network. http://www.fnet.bosai.go.jp/. Accessed 10 Apr 2019

Ogata Y (1988) Statistical models for earthquake occurrences and residual analysis for point processes. J Am Stat Assoc 83:9-27

Ogata Y (1989) Statistical model for standard seismicity and detection of anomalies by residual analysis. Tectonophysics 169:159-174

Ogata Y (1992) Detection of precursory relative quiescence before great earthquakes through a statistical model. J Geophys Res 97:19845-19871

Ogata Y (1999) Seismicity analysis through point-process modeling: a review. Pure Appl Geophys 155:471-507

Ogata Y (2005) Detection of anomalous seismicity as a stress change sensor. J Geophys Res 110:B05S06. https://doi.org/10.1029/2004JB003245

Ogata Y (2006) Statistical Analysis of Seismicity — updated version (SASeis2006), ISM Computer Science Monographs, No. 33, The Institute of Statistical Mathematics, Tokyo, Japan. https://www.ism.ac.jp/ ogata/Ssg/ ssg_softwaresE.html (manual). (manual and codes). Accessed 1 Apr 2014

Ogata Y (2006b) Monitoring of anomaly in the aftershock sequence of the 2005 earthquake of M7.0 off coast of the western Fukuoka, Japan, by the ETAS model. Geophys Res Lett 33:01303. https://doi.org/10.1029/2005G L024405

Ogata Y (2007) Seismicity and geodetic anomalies in a wide area preceding the Niigata-Ken-Chuetsu earthquake of 23 October 2004, central Japan. J Geophys Res 112:B10301. https://doi.org/10.1029/2006JB004697

Ogata Y (2010) Space-time heterogeneity in aftershock activity. Geophys J Int 181:1575-1592. https://doi.org/10.1111/j.1365-246X.2010.04542.x

Ogata Y (2011) Pre-seismic anomalies in seismicity and crustal deformation: case studies of the 2007 Noto Hanto earthquake of M6.9 and the 2007 Chuetsu-oki earthquake of M6.8 after the 2004 Chuetsu earthquake of M6.8. Geophys J Int 186:331-348

Ogata Y, Tsuruoka H (2016) Statistical monitoring of aftershock sequences: a case study of the 2015 Mw7.8 Gorkha, Nepal, earthquake. Earth Planets Space 68:44. https://doi.org/10.1186/s40623-016-0410-8 
Ogata Y, Jones LM, Toda S (2003) When and where the aftershock activity was depressed: Contrasting decay patterns of the proximate large earthquakes in southern California. J Geophys Res 108(B6):2318. https://doi. org/10.1029/2002JB002009

Terakawa T (2014) Evolution of pore fluid pressures in a stimulated geothermal reservoir inferred from earthquake focal mechanisms. Geophys Res Lett 41:7468-7476

Terakawa T, Hashimoto C, Matsu'ura M (2013) Changes in seismic activity following the 2011 Tohoku-oki Earthquake: effects of pore fluid pressure. Earth Planet Sci Lett 365(1):17-24

Tsuruoka H, Ogata Y (2015a) Development of Seismicity Analysis software: TSEIS-ETAS module implementation, 9th InternationalWorkshop on Statistical Seismology (StatSei9), Arcona Hotel am Havelufer, Potsdam, Germany, 17 June 2015. https://statsei9.quake.gfz-potsdam.de/doku. php?id=13_presentations:start. Accessed 1 Sep 2018

Tsuruoka H, Ogata Y (2015b) Development of seismicity analysis tool XETAS, Programme and abstract, the Seismological Society of Japan, 2015, Fall meeting, S09-P01, Kobe, Japan, 26 October

Utsu T (1961) A statistical study on the occurrence of aftershocks. Geophys Mag 30:521-605

Utsu T (1965) A method for determining the value of $b$ in a formula $\log n=\mathrm{a}-\mathrm{bM}$ showing the magnitude-frequency relation for earthquakes. Geophys Bull Hokkaido Univ 13:99-103
Utsu T, Ogata Y, Matsu'ura RS (1995) The centenary of the Omori formula for a decay law of aftershock activity. J Phys Earth 43:1-33

Yoshida K, Hasegawa A (2018) Hypocenter migration and seismicity pattern change in the Yamagata/Fukushima border, NE Japan, caused by fluid movement and pore pressure variation. J Geophys Res 123:6

Yoshida K, Saito T, Urata Y, Asano Y, Hasegawa A (2017) Temporal changes in stress drop, frictional strength, and earthquake size distribution in the 2011 Yamagata-Fukushima, NE Japan, earthquake swarm, caused by fluid migration. J Res Solid Earth, Geophys. https://doi.org/10.1002/2017J B014334

Yoshida K, Hasegawa A, Yoshida T, Matsuzawa T (2018) Heterogeneities in stress and strength in tohoku and its relationship with earthquake sequences triggered by the 2011 M9 Tohoku-Oki Earthquake. Pure Appl Geophys 176:1335-1355

\section{Publisher's Note}

Springer Nature remains neutral with regard to jurisdictional claims in published maps and institutional affiliations.

\section{Submit your manuscript to a SpringerOpen ${ }^{\circ}$ journal and benefit from:}

- Convenient online submission

- Rigorous peer review

- Open access: articles freely available online

- High visibility within the field

- Retaining the copyright to your article

Submit your next manuscript at $\boldsymbol{\nabla}$ springeropen.com 